\title{
Fine dei movimenti e nuove identità generazionali nella narrativa italiana degli anni ottanta: Tondelli e Palandri
}

Fin des mouvements et nouvelles identités générationnelles dans la littérature italienne des années quatre-vingt : Tondelli et Palandri The end of political movements and new generational identities in the narrative of the eighties: Tondelli and Palandri

\section{Ugo Perolino}

\section{(2) OpenEdition}

\section{Journals}

Edizione digitale

URL: http://journals.openedition.org/cei/447

DOI: $10.4000 /$ cei.447

ISSN: 2260-779X

Editore

UGA Éditions/Université Grenoble Alpes

Edizione cartacea

Data di pubblicazione: 15 mars 2012

Paginazione: 151-161

ISBN: 978-2-84310-222-6

ISSN: 1770-9571

Notizia bibliografica digitale

Ugo Perolino, «Fine dei movimenti e nuove identità generazionali nella narrativa italiana degli anni ottanta: Tondelli e Palandri», Cahiers d'études italiennes [Online], 14 | 2012, online dal 15 septembre 2013, consultato il 27 mars 2021. URL: http://journals.openedition.org/cei/447 ; DOI: https://doi.org/ $10.4000 /$ cei. 447 


\title{
FINE DEI MOVIMENTI E NUOVE IDENTITÀ \\ GENERAZIONALI NELLA NARRATIVA ITALIANA \\ DEGLI ANNI OTTANTA: TONDELLI E PALENDRI
}

\author{
Ugo Perolino \\ Università di Pescara
}

Nel tracciare le linee abrasive di una autobiografia della prima Repubblica, Guido Crainz ricorda che l'edonismo degli anni Ottanta fu reso disponibile sul mercato delle rappresentazioni sociali per l'attrazione esercitata da un film, La febbre del sabato sera, emblematico di nuovi stili di comportamento e di aggregazione giovanile. Sulla stessa linea di disinibita trasgressione e di liberazione dai modelli culturali dei "fratelli maggiori" si collocava un libro, Altri Libertini ${ }^{1}$ di Pier Vittorio Tondelli, destinato a diventare il breviario di una o più generazioni di giovani narratori esordienti sulla scena letteraria nel corso del decennio. La politica e l'orizzonte dell'impegno, scrive Crainz, apparivano d'un tratto disertati "per il divertimento, il corpo e la moda» ${ }^{2}$.

Quel fenomeno complesso, che incide oltre l'ambito letterario per l'affermarsi di abitudini e inclinazioni innovative sul piano del costume (significativamente, nell'estate del 1976, Leonardo Vergiani si interroga sulla moda del seno nudo sulle spiagge italiane) ${ }^{3}$, con infrazione di antichi tabù (dopo lo choc prodotto dal referendum sul divorzio), troverà nella

I. Per le opere tondelliane si veda: P. V. Tondelli, Opere, vol. I, Romanzi, teatro, racconti, e vol. II, Cronache, saggi, conversazioni, a cura di Fulvio Panzeri, Milano, Bompiani, 200o. La numerazione delle pagine sarà riferita a questa edizione, mentre le singole opere saranno indicate nelle note in forma abbreviata (AL: Altri Libertini; CS: Camere separate; MSC: Il mestiere dello scrittore). Alcune citazioni sono tratte da P. V. Tondelli, Un Weekend postmoderno, Milano, Bompiani, 2005 (nel seguito richiamato con la sigla WK).

2. G. Crainz, Autobiografia di una Repubblica. Le radici dell'Italia attuale, Roma, Donzelli, 2009, p. I30 sgg. La citazione si trova a p. I3I.

3. Si veda L. Vergani, «Perché il seno nudo sulle spiagge», Corriere della Sera, 7 luglio 1976; successivamente in F. Contorbia (a cura di), Giornalismo italiano 1968-200I, Milano, Mondadori, 2009, pp. 472-475. Il fenomeno del topless, sebbene in qualche modo collegato con la liberazione della sessualità perseguita dai movimenti giovanili, ha connotazioni radicalmente diverse dal nudismo praticato al festival del Parco Lambro del 1976. 
televisione commerciale uno straordinario mezzo di diffusione. Ma prima di essere relegate nella sfera dell'entertainment, le tematiche del personale politico, causa e insieme effetto del disincanto verso la militanza, erano state portate lungamente in gestazione nella vita del movimento.

Si devono retrodatare alla metà degli anni Settanta i primi segni di quella trasformazione critica che sposterà in primo piano - derubricando i miti del marxismo classico, e in primo luogo quello della centralità operaia - il vissuto corporeo, la sessualità, i rapporti interpersonali enfatizzati dal successo di un libro come Porci con le ali, a proposito del quale Giuliano Zincone evidenziava la «massima ironia e massima scioltezza ideologica (nel giardino della sinistra e dell'oggi): Gramsci e Vaneigem, Lenin e Gloriaguida, Tolstoi e le sexy-favole» ${ }^{4}$. Erano interessantissime anche le annotazioni di Zincone su linguaggio e psicologia degli adolescenti, Rocco e Antonia, che «si innamorano durante una manifestazione politica», «si trovano e fanno l'amore», «si isolano nella felicità»: «È incredibile come tutto questo risulti autentico e tremendamente importante - scriveva - in un libro in cui tutti i giovani protagonisti fingono che le cose importanti siano altrove, nelle strutture della società, nella lotta di classe, nei discorsi di Zaccagnini e Berlinguer»`.

Se messo a confronto con le ruvidezze del romanzo della Ravera, Boccalone (1979) di Enrico Palandri sembra restaurare la centralità sentimentale degli spazi collettivi (in primo piano le piazze e i portici di Bologna), per verificarne magari la progressiva erosione, l'inagibilità dopo gli scontri e la repressione del marzo 1977 («Le belle facce che avevo incontrato tutto l'inverno in università, a primavera in piazza maggiore [...] in giugno sono maschere di gesso [...] Bologna è bruciata») ${ }^{6}$, da cui si stacca criticamente la solitudine dell'io narrante («Dovremmo scriverla tutti assieme questa storia, con molte voci confuse assieme, dimenticate sulla pagina scritta: il registratore che adopero io è invece questa maledetta bocca larga, che perde in continuazione») ${ }^{7}$. È stato proprio Tondelli a riconoscere in Boccalone «il libro che ha aperto la strada alla nuova letteratura degli anni ottanta», inaugurando quella «lettura generazionale» che sarà ampiamente discussa, tra gli altri, da Panzeri e dallo stesso Palandri. Nel

Happening carico di significati collettivi (almeno nelle intenzioni), quello del Parco Lambro, in cui il corpo viene usato come un dazebao; cosmetico e autoedonistico, l'altro, indicativo dell' italian lifestyle anni Ottanta. 4. G. Zincone, «Emozioni d'amore e ironia di due adolescenti», Corriere della Sera, I agosto 1976, in

F. Contorbia (a cura di), Giornalismo italiano I968-200I, op. cit., pp. 480-483.

5. Ibid., p. 482.

6. E. Palandri, Boccalone, Milano, Bompiani, 2002, p. 48.

7. Ibid., p. 52. 
romanzo l'azione si situa a ridosso del marzo I977, nei mesi della rivolta creativa, dei carri armati inviati a presidiare le sedi universitarie, dell'assalto della polizia a Radio Alice. La recensione tondelliana coglieva gli aspetti meglio armonizzabili ad una lettura non ideologica: "Boccalone è soprattutto una storia d'amore, prima ancora che di crisi politica, la storia di come un innamoramento possa far scoppiare i propri equilibri, creare intensità nuove» ${ }^{8}$. E ancora, con più scoperto rispecchiamento: «Boccalone è la trascrizione, ora eccitata, ora depressa, ora ironica o addirittura comica, di come un rapporto d'amore vissuto con limpidezza e sincerità possa far crescere, e anche abbandonare quelle cose che prima si definivano come abitudine» ${ }^{9}$. La successiva recensione a La via del ritorno mette a fuoco il doppio movimento inscritto nel romanzo («Un lungo viaggio in treno, con soste, ricordi, emozioni ${ }^{\mathrm{I}}{ }^{\mathrm{O}}$ ) e sembra così indicare la strada di una memoria personale da riconquistare nel grande freddo dell'età adulta, come accade in Camere separate, confermando un rapporto intessuto di attenzione e di parziale identificazione che non elude le differenze.

Nel suo esordio letterario Tondelli si era lasciato alle spalle i riferimenti praticabili per la sua generazione: non ne condivideva le connotazioni ideologiche, il residuo di politicismo. Un rifiuto che si riflette in un racconto come Viaggio, in Altri libertini, dove è ripercorsa la vicenda degli anni Settanta seguendo l'evoluzione sentimentale e l'apprendistato sessuale del protagonista, con la consapevole esclusione di ogni riferimento all'attualità. Le narrazioni tondelliane descrivevano paesaggi e tipi umani della Bassa immersi in un tempo febbrilmente sospeso. La cover del libro era illustrata da una foto di Sven Simon, l'immagine di due giovani autostoppisti in jeans e maglietta ai bordi di una strada; sullo sfondo l'ombra di un camion che si avvicina. Non è necessaria alcuna forzatura ermeneutica per intendere la selva di riferimenti ai miti della beat generation: l'anticonformismo e la fuga dalla quotidianità, una rinnovata libertà dopo la stagione

8. P. V. Tondelli, Enrico Palandri [1979], in WK, pp. 213-216. Il capitolo su Palandri si divide il due parti, due recensioni a Boccalone e a La via del ritorno scritte a distanza di oltre un decennio, rispettivamente nel 1979 e nel 1990, segno di una duratura attenzione da parte di Tondelli, che trova nei romanzi citati, in due momenti diversi della sua esperienza personale e di scrittore, un complesso di situazioni tematiche e narrative analoghe a quelle su cui sta lavorando. La citazione è a p. 213 .

9. Ibid., p. $2 \mathrm{I} 3$.

Io. "Un lungo viaggio in treno, con soste, ricordi, emozioni. Il desiderio di rivedere gli amici dopo dieci anni di volontario esilio in Inghilterra, la paura di trovarli cambiati e indifferenti, il peso del passato, di una militanza politica che ha travolto qualcuno, il sospetto, l'ansia del sentirsene ormai fuori, insieme a un mondo, a rapporti, che sono completamente cambiati con l'evolvere degli anni. L'altro viaggio, innestato in quello principale, da Londra e Edimburgo, è il viaggio della scoperta dell'altro, la nascita dell'amore fra il protagonista, conducente di autobus e la ragazza scozzese che, sullo stesso pullman, fa la hostess.» (Ibid., p. 215) 
del terrorismo e delle stragi, la scoperta di sé, l’iniziazione alla vita, la ricerca della felicità.

Spazio simbolico e crocevia di ogni narrazione, la Via Emilia suggeriva escursioni, avventure, derive, come il grande flume nei racconti di Twain. Nella notte sulla pianura i segni del paesaggio dovevano sconfinare oltre i ristretti significati diurni, in un percorribile altrove:

In tempi non lontani, avrei pensato alla Via Emilia come a una grande città della notte estesa trasversalmente sulla pianura del Po e percorsa, senza interruzione, dai TIR e dalle automobili [...]. Avrei visto allora il grande rullo d'asfalto come una linea di separazione fra la dolcezza della collina emiliana, che di notte s'illumina di fari, bagliori colorati e punti fluorescenti, e l'estesa pianura che affonda verso la foce del Po, con le sue strade che derivano dalla via principale come tanti canali dal letto di un fiume e che portano, anche nelle terre più lontane, quello stesso messaggio d'irrequietezza. (WK, p. 76)

Il carattere del vagabondare tondelliano illuminato dallo «shock intermittente delle city lights», ha scritto Giulio Iacoli, «rimanda a una nuova concezione di flânerie e attraversamento delle località»" ${ }^{\text {II }}$ uno spazio reso più veloce e instabile, fluido, saturo di identità fluttuanti. Da qui quell'aspirazione alla fuga, quella ricerca di luoghi cosmopoliti di cui nutrire l'immaginario, fatta insieme di insofferenze e di nostalgia. Le suggestioni poetiche tondelliane codificano l'irrequietezza, l'assenza di vincoli, la leggerezza come condizione euforica dell'esperienza del reale e della scrittura. Sebbene fossero questi i temi verso i quali si era orientato l'immaginario giovanile nell'onda lunga della contestazione, dal ' 68 in poi, lo scrittore emiliano ne riscopriva il significato prepolitico e l'energia vitalistica. Si tratta di una ispirazione che darà un provvisorio indirizzo alla narrativa italiana degli anni Ottanta, in una traiettoria creativa breve e intensa - da Altri libertini (1980) a Rimini (1985), da Camere separate (1989) fino all' "enciclopedia d'autore» raccolta in Un Weekend postmoderno (1990). La forma simbolica in cui questo sentimento del tempo ha trovato espressione e si è cristallizzato è quella del viaggio come attraversamento di spazi anonimi, esodo, cambio di identità - in un primo momento la via Emilia, la Pianura, i motel e gli autogrill disseminati lungo l'autostrada; poi Rimini e la riviera, i luoghi dello shopping e del turismo di massa; infine le capitali europee, le città globali.

Nei racconti di Altri libertini Tondelli perlustrava una periferia ignorata dalle grandi narrazioni generazionali degli anni Settanta. I suoi scenari

II. Si veda G. Iacoli, Atlante delle derive. Geografie di un' Emilia postmoderna: Gianni Celati e Pier Vittorio Tondelli, Reggio Emilia, Diabasis, 2002, pp. 94-95. 
erano lontani dai centri urbani portatori di storia e identità lungamente sedimentate. «I miei personaggi - racconta in un'intervista a Nico Orengo (TuttoLibri, 9 febbraio 1980) - [...] si muovono in questa prateria, tra Reggio, Modena e Parma [...]. I progetti sono pochi: stare bene, viversi dignitosamente, curare la convivialità». Un universo ai margini della vita collettiva, privo di storia. È sintomatico, ad esempio, come fosse tenue nel suo orizzonte mentale il riferimento a Bologna, dove aveva frequentato il Dams a contatto con personalità importanti per la sua formazione culturale e di uno scrittore come Gianni Celati. In Tondelli, ed è un contrasto ricco di significato, assume centralità e rilievo quel sistema adriatico produttore dell'effimero - l'industria del divertimento, dancing e discoteche che avrebbe trovato la sua capitale in Rimini.

Tondelli apparve subito come l'esponente del nuovo, con le giuste caratteristiche per marcare una discontinuità. Nella fase finale degli anni Settanta entravano in crisi, con la caduta dell'operaismo, culture e pratiche ribellistiche variamente caratterizzate dal rifiuto dell'esistente e da radicali istanze di trasformazione. Non si tratta soltanto della dismissione della lotta di classe o del conflitto generazionale: si registrano invece nuove tendenze, mode, consumi, in cui si afferma il desiderio di sperimentare in modo "gradevole e levigato", cioè privo di asprezze, le seduzioni di una incipiente postmodernità. Nella narrazione tondelliana lo snodo Settanta-Ottanta, il passaggio generazionale e la fine del decennio - che in altre testimonianze assumono i contorni di una drammatica frattura: una "frana" - si esprimono attraverso un'accezione sfumata:

Il fenomeno del "nuovo fumetto italiano" nasce verso la fine degli anni settanta come espressione, senza alcun dubbio, di quella vasta fauna creativa, irridente, dissacrante e non violenta che è passata sbrigativamente alla storia come generazione del I977. Una generazione formatasi culturalmente davanti al teleschermo, cresciuta con in testa il sound delle più belle ballate della storia del rock, diventata giovane maneggiando i paperback e altri gradevoli frutti dell'industria culturale.

Una generazione che, nell'impossibilità di offrire a se stessa una ben precisa identità culturale (seguendo percorsi, ponendosi obiettivi, rivalutando origini), ha preferito non darsene alcuna, o meglio, mischiare i generi, le fonti culturali, i padri putativi, fino ad arrivare alla compresenza degli opposti. Una generazione, e ora lo si vede bene, in cui i linguaggi si confondono e si sovrappongono, le citazioni si sprecano, gli atteggiamenti e le mode si miscelano in un cocktail gradevole e levigato che forse è il succo di questa tanto chiacchierata postmodernità ${ }^{\mathrm{I}}$. 
Questo scivolamento dal '77 al postmoderno era fulmineo, istintivo, ma anche azzerante rispetto all'eredità del recente passato. In un quadro istantaneamente pacificato Tondelli fu percepito come uno scrittore progressista, un prodotto della società civile estraneo al sistema mentale della cultura marxista. Ad un orecchio esercitato l'universo dei suoi personaggi sbandati e stravaganti risultava però aconflittuale e piuttosto versato sui motivi del gioco, del rovesciamento carnascialesco, della trasgressione. Per gli esigenti degustatori del postmoderno, non ignari della lezione bachtiniana e delle risorse polisemiche del comico e del basso corporeo, lo scrittore emiliano risultò innovativo e sperimentale, soprattutto in riferimento al sorvegliato pastiche stilistico-lessicale, ma senza l'intellettualismo tipico delle avanguardie.

A Tondelli si fa credito di avere intuito e formulato coerentemente le prospettive inerenti una condizione giovanile ricca di potenzialità e al tempo stesso esposta a drammatiche involuzioni. Se si prende come modello delle analisi degli intellettuali legati al PCI la distinzione delle due società - da una parte i "garantiti", con al centro la classe operaia, e dall'altra gli studenti - risulta chiaro come tutto quello che nel mondo giovanile conservava qualche elemento di spontaneità dovesse muoversi lontano dal recinto dei partiti. Ma la distanza dagli anni Settanta farà emergere, per contrasto, le ombre del presente, in particolare la perdita di gravità e di peso:

Essere giovani, in quel decennio - si legge in Un Weeekend postmoderno - significò una cosa importantissima: essere presi in considerazione, avere la consapevolezza che il destino della società si giocava (ed era giocato) sulle proprie spalle. I ragazzi erano la "piazza". Fu da questo giovanilismo imperante che nacquero, da un punto di vista esistenziale, le degenerazioni di quegli anni; proprio dal fatto di voler vivere la propria vita (e di essere autorizzati a farlo dalla violenza di stato) come un "assoluto avven-turoso"

Gli elementi di novità introdotti dallo scrittore emiliano non passarono ovviamente inosservati e il libro divenne un caso mediatico. Massimo D’Alema, in una breve nota di lettura per l'Espresso (n. 2, 1980), sottolineava, ad esempio, nel sentimento dell'amicizia, nella «ricerca di un rapporto di solidarietà umana» e nel gusto dell'avventura, i nuclei rilevanti dei racconti tondelliani. Allo stesso tempo evidenziava come Altri libertini fosse un libro "politico", «Se non altro perché l'esperienza giovanile che racconta svela una "mancanza" di politica, o, se si preferisce, una crisi della politica» ${ }^{14}$. Crisi della politica, dunque. Si avvertiva in quelle storie, ricche

I3. P. V. Tondelli, Quel ragazzo... [1985], in WK, pp. I23-I25. La citazione è a p. I25.

14. La contrapposizione tra Boccalone e Altri libertini era articolata in chiave politica. In un libro denso di suggestioni Enrico Palandri scrive che D’Alema «sollecitava in Pier un rientro nel mainstream, cosa che tra 
di riferimenti a precisi modelli letterari (l'Arbasino dell'Anonimo lombardo e, soprattutto, delle Piccole vacanze; poi Burroughs, Bukowskj, Celati, ecc.) un cambio di passo rispetto al protagonismo giovanile del decennio precedente, una sorta di stanchezza, e perciò anche di normalizzazione, alla fine di una lunga fase di scontro generazionale. Allo stesso tempo, ed è questa la ragione del suo successo, Tondelli apriva una fase nuova nella quale i fumetti e il cinema avrebbero contato infinitamente di più del discorso ideologico. D'altro canto i racconti di Altri libertini manifestavano inquietudini innestate nel cuore del modello emiliano, tradizionalmente posto sotto l'influenza del PCI, e rendevano espliciti pericoli e insidie collegati tanto alla crescita economica quanto ai cambiamenti inscritti nella struttura sociale.

In Tondelli la continuità culturale con il movimento del ' 77 risultava in molti punti scheggiata, problematica, interrotta. Lo scrittore tramandava frammenti e ritagli di un linguaggio collettivo che si era im-presso nella memoria di quella generazione attraverso la musica rock. Un personaggio di Altri libertini, Miro, esprime il proprio desiderio attraverso le parole di una canzone di Nico e dei Velvet Underground, I'll be your mirror, che viene recitata come un motivo troppo noto per essere virgolettato; Postoristoro, il racconto che apre il libro, ripete gesti, situazioni, momenti di pura ebbrezza autolesionistica: l'attesa spasmodica del pusher, l'agonia dell'astinenza, tipici di certe ballate di Lou Reed ${ }^{15}$. C'era in questo metalinguaggio pop, colloidale e polifonico, la capacità di agglutinare tutta una massa di materiali largamente diffusi nelle culture giovanili. Tondelli e l'intero movimento del '77, a Bologna e altrove, erano letteralmente innamorati della "tenebrosità dannata» dei Velvet. Erano i simboli della New York urbana e decadente degli anni Sessanta, e di quel mondo di derelitti e freak che popola i film di Warhol. E poi lo affascinava l'omosessualità e la bisessualità di quell'ambiente. Quella della Factory era una cultura individualista, narcisista, alimentata da spinte autodistruttive: l'eros sadomasochistico, la morte per overdose, il «salto nel vuoto». La scrittura dell'ultimo

l'Espresso e Il Corriere per lui è avvenuta, almeno fino a Camere separate». Consultare E. Palandri, Pier. Tondelli e la generazione, Roma-Bari, Laterza, 2005, p. 70.

I5. Nell'articolo Morte per overdose [1986] Tondelli ricorda come «alcune tra le più belle ballate che la storia del rock abbia mai conosciuto, da Heroin, appunto, a Waiting for my Man dei Velvet Underground» abbiano interpretato «le aspirazioni, la rabbia, le delusioni, le tragedie [...] di una o due generazioni che in quei miti si è specchiata, ha creduto, e per far parte di quei miti si è ammazzata». E proseguiva osservando che «Togliere la droga dal rock è togliergli vent'anni di storia e di rabbia giovanili, vent'anni di grandissime interpretazioni musicali e grandissimi testi poetici». P. V. Tondelli, Morte per overdose [1986], in WK, pp. 304-305. La citazione è a p. 305 . 
Tondelli appare capillarmente imbevuta dei motivi della malattia, dell'espiazione, dell'eros sadomasochistico, che trova i suoi archetipi maledetti nei tatuatissimi angeli del male di Morrissey o di Genet ${ }^{16}$.

Questa base espressiva pop e decadente fissa per sempre i confini dell'immaginario tondelliano. L' «universo mitico» degli Smiths, si legge nelle pagine di Un Weekend postmoderno, «appare incluso in quella stagione dell'adolescenza, e della prima giovinezza, avara di piccolezze e ricca invece di difficoltà, di domande non risolte, di angosce, di struggimenti, di conflitti, di passioni, di intensità autodistruttive». Quando, nel 1988, si trovò a scrivere della fine di Andrea Pazienza, forse l'artista più eclettico e geniale del movimento, Tondelli osservò che l'autore delle Avventure di Penthotal aveva incarnato «il lato negativo di una cultura e di una generazione che non ha mai, realmente, creduto a niente, se non nella propria dannazione ${ }^{17}$. In quella morte, che faceva coincidere il destino di Andrea con quello di «tantissimi suoi coetanei», Tondelli vedeva «una grandezza straordinaria, anche se costruita sulle miserie del quotidiano, e una coerenza che solo gli ipocriti possono biasimare». Era il lato tragico e aristocratico di quella cultura della festa che nelle sue inflessioni popolari aveva caratterizzato l'atmosfera degli anni Settanta, ed era esplosa in mille frantumi al festival del parco Lambro del '76, che Gianfranco Manfredi, «pensoso e ironico cantautore del movimento» (Crainz), descrisse come «l'ultimo spettacolo» di quella generazione (la canzone si intitola Un tranquillo festival pop di paura: «È l'ultimo spettacolo non solo della festa / la mia generazione che vuota la sua testa»).

Sebbene vi fossero radici e percorsi comuni, Tondelli si sottrasse a quel contesto generazionale e al collettivismo che ne costituiva il brodo di coltura. In Viaggio, il più ampio e autobiografico dei racconti di Altri libertini, dopo un lungo vagabondare tra le capitali europee - Parigi, Londra, Amsterdam, Berlino - il protagonista trascorre a Bologna le giornate del marzo 1977, ma anziché partecipare all'occupazione dell'università resta in casa, indifferente al corso degli eventi, fisso nel pensiero del «corpo di Michel che sotto terra si decompone e si scioglie» ( $A L$, p. 86). In Camere separate, la cui vena autobiografica risente delle influenze di Peter Handke e di Christopher Isherwood, è descritto con molta precisione il limite, la soglia che il mito tragico dell'adolescenza impone di non attraversare. Lo sguardo, che ha oltrepassato la linea d'ombra, è entrato in un tempo di solitudine e di abbandono. Il confine è quello della vita adulta, con l'assun-

16. P. V. Tondelli, The Smiths [1986], in WK, pp. 300-303.

17. P. V. Tondelli, Andrea Pazienza [1988], in WK, pp. 209-212. La citazione è alle pp. 2II-2I2. 
zione di responsabilità, la stabilizzazione della routine del lavoro, dei legami familiari e sentimentali, l'orizzonte ristretto alle abitudini quotidiane. Leo, il protagonista di Camere separate, a trentadue anni stenta a riconoscersi nel profilo di un viso "appesantito e affaticato» riflesso nell'oblò di un aereo. La sua faccia gli appare «ogni giorno più strana» poiché l'immagine che conserva del proprio volto è «sempre e immortalmente quella del sé giovane e del sé ragazzo». Il suo lento ritorno a casa, in un "piccolo borgo della bassa padana» dove è nato, nella stanza dove ha scritto «le sue prime pagine, i diari, la tesi di laurea», rappresenta un luttuoso pellegrinaggio lungo le stazioni e i luoghi in cui si è consumata una perdita. La malattia e la morte di Thomas, l'essere amato, è in realtà quella soglia, oltre la quale Leo rinasce alla vita adulta.

La società italiana degli anni Ottanta si è offerta allo sguardo tondelliano sotto forma di spettacolo, trasgressione carnevalesca, erotizzata affabulazione di maschere e identità. Lo spazio simbolico che sintetizza metamorfosi e seduzioni del nuovo decennio viene identificato nella riviera adriatica, «una cosmogonia estiva e ferragostana della libido nazionalpopolare». Rimini folcloristicamente appare come una "Nashville patriottica e poliglotta», una "ardente, improvvisata e autogestita carnevalata rabelaisiana» ${ }^{18}$; «Fiabilandia [...] è la riviera adriatica tutta intera: un parco di divertimenti che si definisce in rapporto non tanto ai paesaggi reali, quanto piuttosto ai paesaggi della nostra immaginazione» ${ }^{19}$. Rivelano intelligenza e intuito sociologico anche certe pagine di Un weekend postmoderno dove è raccontato l'ambiente delle corse all'ippodromo del Savio, con la folla vociante "che sa tanto di fauna da Piazza degli Affari», tra "gelatai ambulanti e piadine al prosciutto", un mondo ripreso dalle "sensibilissime antenne delle emittenti private che da tempo diffondono dirette quotidiane dagli ippodromi di Montecatini, Milano, Firenze». Lo sguardo tondelliano è capace di isolare con sorprendente precisione dettagli che rivelano nuovi comportamenti e consumi di massa (la febbre del gioco, il popolo dei piccoli risparmiatori di borsa, lo sviluppo dell'emittenza televisiva privata) che avrebbero trovato soggetti e attori in grado di interpretarne le istanze. Fulvio Panzeri ha indicato in Rimini il punto di arrivo di una ricerca inscritta nei codici del postmoderno "come prova di vari linguaggi e soprattutto di differenti costruzioni narrative, coordinate nel flusso di un viaggio alla scoperta della Riviera romagnola, delle sue mitologie nazionalpopolari, di tutto quel kitsch da spiaggia e da discoteca», che

I8. P. V. Tondelli, Adriatico Kitsch (1982), in WK, pp. 98-IoI.

I9. P. V. Tondelli, Spiagge (I986), in WK, pp. II2-II6. La citazione è a p. II4. 
offre a Tondelli un "palcoscenico collettivo», lo «scenario postmoderno a lungo inseguito ${ }^{20}$. Secondo Enrico Palandri, al contrario, l'interesse verso la dimensione dell'effimero, tributo all'effervescenza del decennio, induce Tondelli a verticali cadute di tensione da cui si riscuote con la ricerca di nuovi e più forti valori letterari, ricerca documentata dall'approdo di Camere separate. La "grande differenza", annota Palandri sul filo di un confronto che è anche autobiografia generazionale, «mi pare proprio l'atteggiamento verso la moda. Per Pier, che è meno severo e forse meno segnato dal cambiamento d'epoca, la moda è allegria, mobilità sociale, feste [...]. Per me e altri la moda è, leopardianamente, sorella della morte» ${ }^{21}$.

Il vitalismo agisce in Tondelli come antidoto alle pulsioni autodistruttive e conferisce alle sue narrazioni una curvatura progressiva, rivolta invariabilmente al futuro. Lo scrittore di Correggio difese coerentemente gli anni Ottanta, "che molti già si affrettano incautamente a vituperare», ricordando che in quel decennio erano emersi «valori e comportamenti assolutamente non trascurabili». Innanzitutto «un ritorno alla solidarietà - non più di classe, ma generalmente civile - di fronte alle emergenze sociali, come l'emigrazione dai paesi extracomunitari, la criminalità mafiosa, i sequestri di persona, la cura e il recupero dei tossicodipendenti». La coscienza giovanile, condensata simbolicamente nei grandi raduni rock, aveva assunto come tema centrale $\mathrm{i}$ «non più rimandabili problemi ecologici del pianeta» ${ }^{22}$.

La narrativa degli anni Ottanta (spesso dichiaratamente tondelliana, o post-tondelliana, anche per l'azione di talent scout svolta dallo scrittore emiliano) è alimentata dalla volontà di esplorare spazi e forme della contemporaneità e di recuperare la forma chiusa del racconto. Una volta esaurita la spinta vitalistica, l'ingresso nell'età adulta torna ad assumere i tratti di una caduta nella quotidianità deludente e opaca. Nei racconti di Claudio Piersanti, in particolare nella raccolta L'amore degli adulti (1989), il minimalismo dei conflitti personali, l'impermanenza dei sentimenti, il clima di generale svuotamento delle tensioni collettive, fanno emergere, per contrasto, inquietudini altrimenti sepolte nelle profondità della provincia italiana. È una generazione silente, di trentenni già senilmente segnati, quella raccontata da Piersanti; una generazione resa più ansiosa dallo spettro del declino economico, un argomento che entrerà nel dibattito politico soltanto più tardi ma già presente alla fine di un decennio

20. F. Panzeri, Pianura progressiva, introduzione a Opere, Romanzi, teatro, racconti, op. cit., pp. IX e X.

2I. E. Palandri, Pier. Tondelli e la generazione, op. cit., pp. 86-87.

22. P. V. Tondelli, Anni Ottanta, in MSC, pp. 84I-843. La citazione è a p. 84I. 
caratterizzato dall'esplosione del debito pubblico e dall'altissimo livello di corruzione politico-economica che inquina il sistema dei partiti. Nel solco di questa scrittura del disincanto e della quotidianità - e, pertanto, anti-tondelliana - sono da registrare, accanto a Piersanti, i libri di Palandri, soprattutto il romanzo La via del ritorno, che prospetta un ripensamento critico dell'esperienza giovanile e che fu definito da Tondelli come «una ballata sul tempo, sulle persone che si perdono, su quelle che restano nella nostra coscienza» ${ }^{23}$. E Giulio Mozzi, autore di narrazioni scabre che spingono il minimalismo stilistico verso esiti di acuta e dolorosa afasia (Questo è il giardino, 1990); Claudio Lolli, attivo come cantautore nel ' 77 bolognese e autore di una raccolta di racconti, Giochi crudeli, pubblicata nel 1993 da Transeuropa, che aveva prodotto le antologie tondelliane. E il 1993 è anche l'anno di esordio del marchigiano Angelo Ferracuti con una raccolta di racconti, Norvegia (1993), profondamente influenzati dalla lezione carveriana e da Celati.

Ma c'è un secondo Tondelli che Panzeri indica esemplarmente nel racconto Pier a gennaio (1986), che modellizza la tematica del ritorno a casa, da Biglietti agli amici fino a Camere separate. Un Tondelli malinconico, introspettivo ed elegiaco e perciò portato a insistere sui codici formali dell'autobiografia (Ingeborg Bachmann, Peter Handke, Christopher Isherwood). Uno scrittore orientato verso una maturazione, non si sa quanto compiuta e tecnicamente meditata, che si lascia alle spalle i contenuti mitici dell'adolescenza. La fenomenologia dell'abbandono allude a questa separazione non solo dall'essere amato, ma da se stessi e da ciò che si è stati. Una volta scontate novità e rotture generazionali, il percorso tondelliano era esposto a pericolose involuzioni, su cui il giudizio resta necessariamente sospeso ${ }^{24}$. A riprova del fatto che una valutazione obiettiva dell'opera tondelliana non può essere disgiunta da una prospettiva complessiva sugli anni Ottanta, di cui l'autore di Camere separate intuisce il declino come un fatto intimo e irrevocabile.

23. P. V. Tondelli, Enrico Palandri [1990], in WK, p. 216.

24. Involuzioni e ricadute neosentimentali che Alberto Arbasino, mai tenero con lo scrittore emiliano, ha ironicamente evidenziato: «Ma se Tondelli mi avesse chiamato, ci sarebbero stati almeno due temi "scomodi" su cui discutere. Il riaffacciarsi, dopo anni e anni di Epico, di Crudeltà, di Engagement, di Strutturalismo, di Semiologia, eccetera, di un intimismo sentimentale ove il suo tocco aveva una grazia molto singolare e molto tenera, ma che stava lì lì per dilagare in una inflazione neopiccoloborghese di piccoli dispiaceri e piccole crisi di intellettuali e di coppie nelle meschinità di un quotidiano "minimal".» (A. Arbasino, "Tondelli al telefono", la Repubblica, 26 ottobre 200I) 
